Bundesgesundheitsbl 2015 · 58:638-640

DOI 10.1007/s00103-015-2151-4

Online publiziert: 14. April 2015

๑) Springer-Verlag Berlin Heidelberg 2015

Bekanntmachung des Umweltbundesamtes

\title{
Aufgabenprofil und Zusammensetzung der Kommission Human-Biomonitoring (HBM-Kommission) für die Jahre 2013-2016
}

1. Die Ableitung von toxikologisch/ epidemiologisch begründeten $\mathrm{Be}$ urteilungswerten (HBM-I- und HBM-II-Werte) zur gesundheitlichen Bewertung der Stoffbelastung der Allgemeinbevölkerung, also der nicht beruflich belasteten Bevölkerung.

Für die Ableitung von Human-Biomonitoring (HBM)-Werten hat die HBM-Kommission mit dem neuen Grundsatzpapier [1] drei Wege beschrieben. Die Ableitung kann demnach zum einen auf der Basis von Studienergebnissen erfolgen, die einen Zusammenhang zwischen der Konzentration eines Stoffes oder seiner Metaboliten in menschlichen Körperflüssigkeiten und dem Auftreten adverser Wirkungen belegen. Zum anderen können zur Ableitung aus Tierversuchen ermittelte, toxikologisch begründete tolerable Aufnahmemengen oder kritische Effekte herangezogen werden. Im zuletzt genannten Fall wird neben dem NOAEL (No Observed Adverse Effect Level) oder dem LOAEL (Lowest Observed Adverse Effect Level) nach Möglichkeit auch das BenchmarkVerfahren zur Ermittlung von toxikologischen Kenngrößen wie der BMD (Benchmark Dose) und der BMDL (Benchmark Dose Lower bound - einseitige, untere $95 \%$-Vertrauensgrenze der BMD) verwendet. Notwendige Zeitextrapolationen, Inter- sowie Intra- speziesunterschiede u.a. werden mittels sog. Assessmentfaktoren berücksichtigt. Für den in praxi häufigsten Fall des Bezugs der experimentellen Daten auf eine äußere Dosis ist eine Umrechnung auf die innere Exposition erforderlich. Die HBM-Werte als Stoffkonzentrationen in Körperflüssigkeiten beziehen sich dabei auf die Angabe einer äquivalenten inneren Dosis im Expositionsgleichgewicht. Für die Umrechnung der äußeren Exposition (Dosis) in eine innere Exposition werden neben toxikokinetischen Dosis-Konzentrations-Beziehungen beim Menschen auch PBPK (Physiologisch Basierte Pharmakokinetische)-Modelle als geeignete Verfahren angesehen, wenn sich die Modelle als valide erwiesen haben. Vor dem Hintergrund, dass die Bevölkerung einer Vielzahl von Stoffen ausgesetzt ist, von denen viele den gleichen Wirkmechanismus aufweisen (z. B. bestimmte Phthalate), ist häufig eine Gruppen- statt Einzelstoffbewertung erforderlich. Die HBMKommission hat es sich zur Aufgabe gemacht, hierzu entsprechende Vorschläge zu unterbreiten.

2. Die Ableitung von Referenzwerten mittels statistischer Verfahren zur Beschreibung der Grundbelastung der Bevölkerung und deren zeitlicher Entwicklung. Die Referenzwerte werden statistisch aus dem 95 . Perzentil der Belastung einer Stich- 
probe berechnet. Bevorzugt werden sie aus bevölkerungs-repräsentativen Daten der Deutschen Umweltstudie zur Gesundheit GerES (früher Umwelt-Survey genannt) abgeleitet. Referenzdaten sind für die Überwachung, für eine bundesweit einheitliche Einschätzung von Daten zur korporalen Schadstoffbelastung sowie für die Einordnung von Ergebnissen individueller Situationen, unfallbedingter Expositionen und anlassbezogener Untersuchungen unerlässlich. Da sie statistisch abgeleitet sind, liefern sie keine Bewertung gesundheitlicher Risiken. Abhängig von den jeweiligen Stoff-Eigenschaften kann einerseits die durchschnittliche Belastung bereits im toxikologisch relevanten Bereich liegen, andererseits müssen überdurchschnittliche Belastungen keinerlei Wirkungen auslösen.

Für Substanzen, für die keine Wirkschwelle existiert oder für die aus ethischen Gründen keine Wirkschwelle abgeleitet werden kann, gibt der Referenzwert Informationen darüber, ob eine Belastung über der Hintergrundbelastung liegt.

3. Verknüpfung von HBM-Untersuchungen/-bewertungen mit den Erfordernissen des Vollzugs von Stoffgesetzen sowie der Vorbereitung regulatorischer Maßnahmen. Ob gesetzliche Vorgaben der Chemikalienregulierung greifen oder weitergehende Maßnahmen erforderlich sind, kann mittels HBM nur dann geprüft werden, wenn auch die Stoffe bestimmt werden, die für die Gesundheit der Bevölkerung bedeutsam sind. Bisher wurde die Auswahl der Chemikalien, die in HBM-Projekten untersucht und bewertet wurden, nicht unwesentlich durch die Verfügbarkeit der chemisch-analytischen Methoden bestimmt. Dieser Praxis wird nun durch ein Stoff-Priorisierungs-Verfahren mit anschließender Methodenentwicklung entgegengewirkt $[2,3]$, das sich in erster Linie an der toxikologischen/gesundheitlichen Bedenklichkeit von Stoffen und der Expositions-Wahrscheinlichkeit der Bevölkerung orientiert. Als „Wächter“ für umweltpolitische und regulierende Maßnahmen muss HBM bevorzugt Belastungen mit solchen Stoffen erfassen, die heute präsent sind, produziert, verwendet und vom Menschen aufgenommen werden. Die HBMKommission gibt ggf. Empfehlungen zum Schutz vor gesundheitsbedenklichen Belastungen und dazu, welcher Handlungsbedarf besteht, um Umweltbelastungen zu vermeiden.

4. Beratung bei der Planung und Durchführung von HBM-Studien, bei der Ermittlung des Forschungsbedarfs im Bereich HBM sowie bei speziellen und aktuellen Fragen des HBM in der Hygiene, Umwelttoxikologie und -medizin. Hier steht die Beratung des Umweltbundesamtes bei der Umsetzung des Konzeptes zur gesundheitsbezogenen Umweltbeobachtung im Vordergrund.

5. Auseinandersetzung mit ethischen Aspekten des HBM und Kommunikation dieses Themas gegenüber der Öffentlichkeit.

6. Verstärkte internationale Kooperation zum gegenseitigen Wissenstransfer im Bereich HBM sowie zur vergleichenden Betrachtung unterschiedlicher Bewertungsverfahren.

Mitglieder der HBM-Kommission sind Wissenschaftlerinnen und Wissenschaftler, Fachleute aus Bundes- und Landesbehörden, Universitäten, Kliniken und Forschungseinrichtungen, die aufgrund ihrer fachlichen Kompetenz von dem Präsidenten/der Präsidentin des Umweltbundesamtes für jeweils drei Jahre berufen werden (zuletzt 2013). Die Kommission ist immer interdisziplinär besetzt.

\section{Zusammensetzung der HBM-Kommission}

Vorsitzender: Prof. Dr. Jürgen Angerer Institut für Prävention und Arbeitsmedizin der Deutschen Gesetzlichen Unfallversicherung, Ruhr-Universität Bochum (IPA)

Stellvertretender Vorsitzender: Prof. Dr. Michael Wilhelm

Institut für Hygiene, Sozial- und Umweltmedizin, Ruhr-Universität Bochum
Prof. Dr. Hermann Fromme

Bayerisches Landesamt für Gesundheit und Lebensmittelsicherheit, München

\section{Prof. Dr. Thomas Göen}

Institut und Poliklinik für Arbeits-, Sozial- und Umweltmedizin der Universität Erlangen-Nürnberg

\section{Dr. Birger Heinzow}

Landesamt für soziale Dienste SchleswigHolstein, Dezernat 34: Umweltbezogener Gesundheitsschutz, Kiel

\section{PD Dr. Jürgen Hölzer}

Institut für Hygiene, Sozial- und Umweltmedizin, Ruhr-Universität Bochum

\section{Prof. Dr. Claudia Hornberg}

Fakultät für Gesundheitswissenschaften, Universität Bielefeld

\section{Prof. Dr. Wilhelm Huisinga}

Institut für Mathematik - Wissenschaftspark, Universität Potsdam

\section{Dr. Julia Hurraß}

Gesundheitsamt, Infektions- und Umwelthygiene der Stadt Köln

\section{Dr. Holger M. Koch}

Institut für Prävention und Arbeitsmedizin der Deutschen Gesetzlichen Unfallversicherung, Ruhr-Universität Bochum (IPA)

\section{Prof. Dr. Andreas Kortenkamp}

Institute for the Environment, Brunel University, London

\section{Dr. Inge Mangelsdorf}

Hannover

\section{Dr. Michael Schümann \\ Freie und Hansestadt Hamburg}

\section{PD Dr. Wolfgang Völkel}

Bayerisches Landesamt für Gesundheit und Lebensmittelsicherheit, München

\section{Ständige Gäste}

Arbeitsgemeinschaft der Obersten Landesgesundheitsbehörden (AOLG) -

Arbeitsgruppe „Umweltbezogener Gesundheitsschutz“: Dr. Martin Kraft (Lan- 
desamt für Natur, Umwelt und Verbraucherschutz NRW, Recklinghausen)

Bundesministerium für Gesundheit: Dr. Ute Winkler

Bundesministerium für Umwelt, Naturschutz, Bau und Reaktorsicherheit: Dr. Birgit Wolz

Bundesinstitut für Risikobewertung: Dr. Ulrike Pabel

Robert Koch-Institut: Dr. Hildegard Niemann

\section{Geschäftsführung und Kontakt}

Ansprechpartner für Fragen zur HBMKommission des UBA ist das Fachgebiet II1.2 „Toxikologie, gesundheitsbezogene Umweltbeobachtung", Frau Petra Apel, Email: petra.apel@uba.de

Weitere Informationen und bisherige Publikationen sind unter http://www.umweltbundesamt.de/themen/gesundheit/ kommissionen-arbeitsgruppen/kommission-human-biomonitoring

bzw. http://www.umweltbundesamt. de/en/topics/health/commissions-working-groups/human-biomonitoringcommission abrufbar.

\section{Literatur}

1. Kommission Human-Biomonitoring des Umweltbundesamtes (2014) Grundsatzpapier zur Ableitung von HBM-Werten Stellungnahme der Kommission Human-Biomonitoring des Umweltbundesamtes. Bundesgesundheitsbl Gesundheitsforsch Gesundheitsschutz 57 (1):138-147. doi:10.1007/s00103-013-1867-2. http://link.springer.com/article/10.1007/s00103-013-1867-2, http://www.umweltbundesamt.de/sites/default/files/medien/377/dokumente/art_10.1007_ s00103-013-1867-2-2.pdf

2. Bundesministerium für Umwelt, Naturschutz, Bau und Reaktorsicherheit (BMUB) (2014) Bundesumweltministerium und Chemieverband setzen neue Ziele für Human-Biomonitoring. Pressemitteilung Nr. 118/14. www.bmub.bund.de/N50952/

3. Gemeinsame Pressemitteilung des Bundesministeriums für Umwelt, Naturschutz und Reaktorsicherheit, Verbandes der chemischen Industrie (2013) Bundesumweltministerium und Chemieverband arbeiten bei Human-Biomonitoring weiter zusammen. Nr. 076/13. www.bmub.bund.de/ N50093/ 